\title{
A new trans-Atlantic route structure for strategic flight planning over the NAT airspace
}

\author{
Imen Dhief* ${ }^{\dagger}$, Nour Houda Dougui*, Daniel Delahaye ${ }^{\dagger}$, Noureddine Hamdi* \\ *Heterogeneous Advanced Networking and Applications (HANA) Research group ENSI, Manouba, Tunisia \\ ${ }^{\dagger}$ Laboratory of Applied Mathematics, Informatics and Automatics for Aviation (MAIAA) ENAC, Toulouse, France
}

\begin{abstract}
Air traffic across the North Atlantic airspace has witnessed an incessant increase over the last decades. However, the efficiency of trans-Atlantic air traffic management is still low nowadays due to the limited radar coverage. The availability of Automated Dependent Surveillance-Broadcast systems represents an opportunity to enhance the strategic flight planning over the oceans by reducing the separation standards. Besides, oceanic flights are subject to very strong winds. Flying wind-optimal route yields to a significant fuel and time savings.

In this paper, we propose a new trans-Atlantic route structure that benefits from the jetstreams in order to construct windoptimal flight trajectories. Then, we introduce an optimization model for detecting and resolving conflicts. The analysis is carried out on real traffic data to prove the efficiency of the developed method. Experimental findings show that our approach provides encouraging results in terms of conflict resolution and induced delays.
\end{abstract}

\section{INTRODUCTION}

The North Atlantic airspace (NAT) accommodates air traffic between Europe and North America. It is considered as the second largest oceanic airspace, after the Pacific airspace, and it is the busiest one in the world. In 2012 about 460,000 flights crossed this airspace [1]. Due to increasing passenger demand, time zone differences, jet streams, this vast airspace becomes very congested, especially in peak hours. Currently, the major means of surveillance applied on the oceanic airspaces is the High Frequency (HF) voice Positions (POSs) according to which aircraft have to communicate their positions and specific data to the Oceanic Area Control Center (OACC). However, poor propagation conditions and communication equipment failures often lead to degradation of this communication. Therefore, controllers prefer rather to apply strategic traffic planning in order to overcome the surveillance difficulties. Besides, aircraft operating on the NAT airspace are subject to very strong winds caused by the presence of jet streams. These streams are fast flowing air currents running mainly from west to east. They typically run between 20,000 and 50,000 feet with a speed around $100 \mathrm{kts}$ and can reach $200 \mathrm{kts}$. Their width is relatively narrow compared to their length. As a result, air traffic in the NAT airspace is divided into two major flows: westbound flow, travelling from Europe to North America in the morning and eastbound flow travelling from North America to Europe in the evening. Normally, eastbound flights exploit the jet streams in order to benefit from strong tailwind, while westbound flights would rather avoid the jet streams and stay away from headwinds. In order to overcome all these constraints, namely the lack of surveillance and jet streams, a structure of routes, called
Organized Track System (OTS), was established in the north Atlantic airspace. These routes are daily constructed to satisfy as much as possible the optimal flight profiles. Besides, aircraft are required to apply very restrictive separation standards. Currently, the oceanic norms of separation are higher than the continental one because of the lack of radar coverage over oceanic area. These very demanding separation standards limit the efficiency of the oceanic airspace. Considering these issues, a new kind of communication system, called Automatic Dependence Surveillance-Broadcast (ADS-B), has been recently introduced. Thanks to the accuracy of this system, the oceanic standard separation will be relaxed. Currently, ADSB systems use is increasing around the world. For instance, applying the ADS-B is becoming mandatory for all aircraft operating on the European, Canadian and Australian airspaces since 2015. Moreover, the US has near-term plans to mandate its deployment by 2020 [2]. Besides, statistics showed that, by $2020,95 \%$ of aircraft operating on the NAT airspace will be equipped with ADS-B systems (or equivalent) [1]. Therefore, supposing that all aircraft are equipped with ADS-B is a conceivable assumption which can turn to a fact in the near future.

Our objective, in this work, is to substitute the OTS system while insuring an optimal management of the traffic. The main idea is to take advantage from both the application of ADSB systems, and the exploitation of the jet streams in order to construct wind optimal routes. Thus, we consider only eastbound flights that can benefit from the jet streams. In fact, several researches showed that travelling wind optimal routes have a great positive effect on fuel consumption. For this reason, we propose to merge the OTS tracks on the jet streams. At the same time, we suppose that all aircraft are equipped with ADS-B system so that we can profit from a significant reduction on the separation standards. The most important gain, to be reached by applying this new structure of routes, is to put as many flights as possible on the jet. Therefore, these flights can follow near wind-optimal routes. Thus, we propose, in this paper, a new structure of routes over the NAT airspace that takes advantages from the jet streams. Then, we propose a method to optimize the strategic flight planning using this oceanic route structure.

The rest of this paper is organized as follows: The next section describes the actual route structure on the NAT airspace. Section III contains relevant works on optimizing air traffic over the oceanic airspace. Section IV presents the problem formulation. We mainly describe the new developed route 
structure and present the flight model that we adopt. Section V exposes the methodology of conflict detection and resolution. Computational results are presented in section VI. Finally, we conclude in section VII.

\section{BACKGROUND}

In this section, we present a theoretical background related to our research topic. First, we describe the OTS routes, which represents the actual route structure in the NAT airspace. Then, we introduce a brief description of the ADS-B system and we expose the potential benefits behind its deployment.

\section{A. The Organized Track System}

In order to ensure safe flights over the NAT airspace, the OTS system is established. It is a set of trans-atlantic flight routes that links the Northeast of America to Western Europe. OTS includes typically from 5 to 7 parallel or nearly parallel tracks in each direction (eastbound and westbound) between the altitudes of 29,000 and 41,000 feet. Around 10 waypoints are planned in each track. The tracks are constructed, daily, to take into account the shifting of west-east jet streams. Aircraft operating over the NAT airspace have to satisfy vertical, lateral and longitudinal separation. The OTS system is constructed to satisfy these separation norms. Indeed, the tracks are separated by $60 \mathrm{NM}$ laterally and 1000 feet vertically (the actual separation norms). Thus, once the aircraft are on the OTS

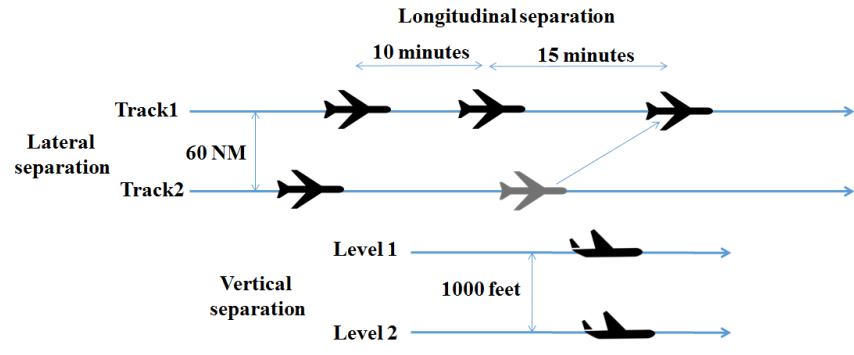

Fig. 1: Separation standards in oceanic airspace

route structure, controllers have only to ensure the longitudinal separation between them. In fact, the longitudinal separation is calculated in terms of time and represents the time between two consecutive flights following the same track. Thus, the longitudinal separation is 10 minutes between two consecutive aircraft following the same track. If an aircraft changes its track, the longitudinal separation becomes 15 minutes with the flights in the new track (Figure 1). As illustrated in figure 2 , the OTS can be represented by a grid of three axes $N_{x}, N_{y}$ and $N_{z}$ :

- Axe $N_{x}$ : Labelled from 1 to $N_{x}$ oriented to the east, it contains a set of nodes representing the waypoints in each track.

- Axe $N_{y}$ : Labelled from 1 to $N_{y}$ oriented to the north, it represents the number of OTS tracks.

- Axe $N_{z}$ : Labelled from 1 to $N_{z}$ started from the lowest.

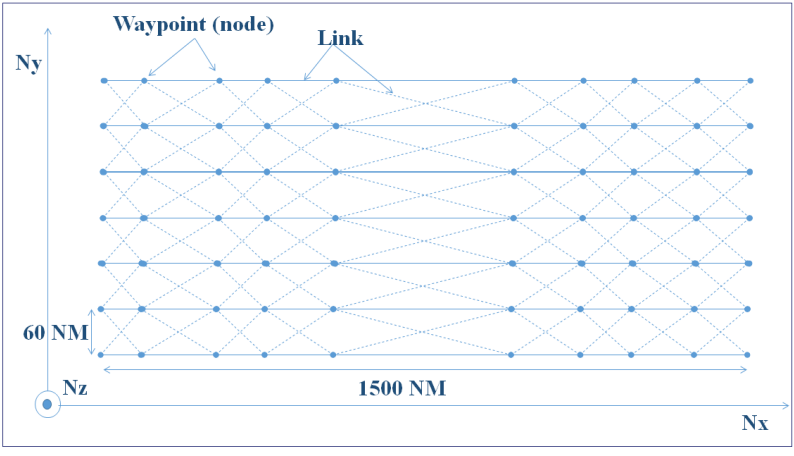

Fig. 2: OTS model

Although there is no radar over the ocean, oceanic controllers must still make sure all the aircraft are conducted safely. Since they can not see the aircraft, controllers rely on pilots to report their position at regular intervals. A position report provides an aircraft's location, speed, and altitude. Pilots used to apply High Frequency Voice Positions to communicate their reports to oceanic control centers. Actually, on most occasions, when pilots communicate with Oceanic Air Traffic Control Centres (OATCC), they do not talk directly to controllers. An international flight service station (IFSS) is responsible to relay messages between aircraft and OATCC. Such units are not always co-located with an OATCC. Besides, it is important to note that controller workload is usually high. Thus some delays can be expected for responses to requests for a change of flight level, route, etc. Besides, we note that depending on atmospheric conditions, it can be relatively noisy with the signal in and out. For these reasons, oceanic controllers usually deny rerouting from one track to another inside the OTS [1]. Thus, flights are more likely to keep the same track from the entrance to the exit of the OTS, and re-routing is rarely authorized.

\section{B. ADS-B systems}

The availability of ADS-B represents an opportunity to enhance air traffic management over the NAT airspace. ADS$\mathrm{B}$ is an airborne-based system that relies upon automatic position reports in order to provide both pilots and controllers with more accurate and reliable information. Based on the principle of providing a global coverage, the ADS-B system ensures a periodic transmission of the aircraft information (position, velocity and heading), via a broadcast data link, to both the controllers and the surrounding traffic. Thanks to the accuracy of this surveillance tool, the air traffic situation will be improved in the oceanic airspace. Thus, a significant reduction in the separation norms can be applied. Longitudinal separation between two aircraft in the same track becomes 2 minutes, instead of 10 minutes when the two aircraft are consecutive on the same track, and 3 minutes instead of 15 minutes, if an aircraft changes its track. Further informations and details about the fonctionalities of ADS-B systems are given in $[3,4]$ 


\section{RELATED WORK}

In the literature, several works deal with the problem of improving aircraft situation over the oceanic airspace. They focus on optimizing flight routes by re-routing the aircraft inside the OTS structure. Each flight is represented by several parameters, such as entry and exit tracks, flight level and Mach number. Once inside the OTS tracks, a flight can request to change some of its parameters. This change can be a re-routing from the initial track, switching to another flight level or varying the Mach number. In [5], authors assumed that all aircraft are equipped with ADS-B systems. Thus, the reduced separation standards can be applied. Under these conditions, aircraft can regularly change tracks. OTS structure is represented by a grid of nodes and links. Each aircraft has the possibility to change its track only on nodes by moving one track up or down. The Genetic Algorithm (GA) is applied to find the optimal flight paths while avoiding conflicts. The same authors applied the Simulated Annealing algorithm (SA) with the same model to resolve the same trajectory optimization problem in [6]. The application of the two algorithms shows that operating the ADS-B system can remarkably improve the traffic situation. Besides, both algorithms can reach a conflict-free solution for real traffic data while optimizing a specific objective function. However, the SA algorithm gets better solutions than the GA algorithm for the considered problem, in much less time. Another work [7] aims to optimize the NAT air traffic by removing the OTS structure and using nearly direct flight routes instead. The idea is to apply the flocking boid model in order to construct a full swarm behavior while assuming that all aircraft are equipped with ADS-B systems. The flight trajectories are represented as a set of discrete points. Initially, each flight cap is steered to its destination point. Then, in each time sample, the flight cap is adjusted using the boid flocking model. Actually, the flocking model represents the form of collective behavior of a large number of interacting agents. It relies upon three heuristic rules. First, the separation rule which guarantees a separation distance between agents, hence, it permits to avoid conflicts. Second, the cohesion rule which is used to ensure the swarm behavior. Finally, the alignment rule which is used in order to maintain agent trajectories quasi-parallel. Besides, considering the specificities of the problem, two additional rules have been included, namely, a force to oblige the flight to reach its destination and a force to avoid the oscillation of the trajectories. The SA algorithm is applied in order to find the optimal balance between the different forces in each time sample. The computational results prove a considerable reduction in the number of conflicts with reasonable delay and elongation from the direct path. Besides, several works consider the aircraft trajectories optimization problem in presence of wind in both continental and oceanic airspace. [8, 9] focus on air traffic optimization in the continental airspace. Concerning the oceanic airspace, many studies treat the NAT airspace. Typically, they consider only eastbound traffic in order to exploit the jet stream tail- winds. [10] evaluate the potential benefits from flying windoptimal routes in the NAT airspace. It shows that a significant gain can be reached when aircraft follow wind-optimal paths regarding cruising time and fuel saving. Another study [11] developed an algorithm that optimizes Trans-Atlantic flight trajectories in presence of winds. The process is divided into two stages. First, the optimal vertical profile for each aircraft is calculated. Then, optimal aircraft headings considering the wind on multiple flight levels are determined. As for the previous study, simulation results allow to conclude that traveling wind-optimal trajectories with optimal vertical profile save time and fuel. However, these wind-optimal routes generate a large number of potential conflicts between flights. In fact, for the two aforementioned works, the conflict detection and resolution problem is not considered. They focus only on optimizing one flight trajectory without considering the totality of the traffic. Some recent works aim to de-conflict windoptimal routes over the oceanic airspace. For instance, both studies $[12,13]$ introduce strategic methods that detect and resolve conflict of wind-optimal flight trajectories in the NAT airspace. In [12], de-conflicting wind-optimal routes were ensured with a SA algorithm combined with local gradient search. The potential number of conflicts was reduced by a small adjustment in departure times and rerouting. In [13], the conflict resolution is insured by a SA algorithm and is based on two maneuvers: changing the departure time, and slightly modifying the geometrical shape of the trajectory while remaining wind-optimal. Computational results show that an interesting reduction in the number of conflicts can be reached. Nevertheless, this method of wind-optimal trajectories is not robust regarding the change of meteorological conditions. In fact, when taking into account uncertainties in wind data, new conflicts appear and the proposed method does not provide solutions to this problem. Moreover, [14] represents the first study that discuss the potential benefit from traveling wind-optimal routes instead of using the Central East Pacific (CEP) airspace route structure. A backward recursion dynamic programming algorithm is used to calculate windoptimal flight routes. The same authors treat the problem of conflict detection and resolution of the wind-optimal routes in [15]. The idea is to strategically schedule flights while ensuring that the interaction between trajectories is manageable. The problem was modeled as a job shop scheduling issue. It has been solved via 0-1 integer programming model. Simulation results prove that the annual economic benefit for airlines companies range between $\$ 3.4$ million and $\$ 8.5$ million if flights follow wind-optimal routes on the CEP. However, it is obvious that flights which follow preferred routes and override the established route structure will never follow in practice the exact pre-determined paths and predicted conflicts may never happen. Therefore, a realistic solution to manage oceanic air traffic necessarily passes through a route structure.

In our study, we focus on the eastbound strategic flight planning. We combine two paradigm in order to benefit from the advantages and strengths of each one. First, we keep the route structure in order to overcome the surveillance coverage 
problem, leading to a more reliable and safe traffic over the NAT airspace. Second, we adjust the route structure to the jet streams direction in order to obtain approximate wind-optimal trajectories.

\section{PROBLEM FORMULATION}

We consider a set of $N$ eastbound flights. Each flight is represented by a set of parameters which are:

- Entry and exit track,

- Track entry time,

- Flight level at waypoints,

- True airspeed in knots.

Some of these parameters are fixed data that we do not alter for the entire problem resolution. While other parameters can be relaxed and represent the variables of our optimization problem. Indeed, we assume that the aircraft speed is constant for the entire trajectory. This assumption is conceivable since we are tackling only the en-route phase. On the other hand, it's important to extend the state space in order to guarantee conflict-free trajectories. Thus, entry delay less than 20 minutes is allowed. Moreover, the entry and exit tracks can be relaxed by allowing aircraft to enter and/or exit an adjacent track. Actually, we mainly do not prefer to change the vertical profile of the aircraft, since it guarantees the optimal fuelconsumption. Nevertheless, we permit to change the requested flight level at waypoints as a last alternative when no conflictfree solution exists. This modification of flight level is restricted to aircraft climb. Descent is not allowed. Besides, changes of entry/exit flight level are not allowed in order to meet as much as possible airline companies' preferences. The entry data of our model are represented below:

- Track $_{\text {In }} \in 1,2, \ldots, N y$ the desired entry track

- Track $_{\text {Out }} \in 1,2, \ldots, N y$ the desired exit track

- $T_{\text {In }}$ the entry time

- $F L_{i} \in 1,2, \ldots, N z$ where $i \in 1,2, \ldots, N_{x}$, the flight level at each waypoint expressed in feet. The distance between each two consecutive flight level is equal to 10 feet.

In order to represent the relaxation that we permit in some flight's parameters, we define the following decision variables:

- ATrack $_{\text {in }}=$ Track $_{I n}+/-1$ the assigned entry track.

- ATrack $_{\text {out }}=$ Track $_{\text {Out }}+/-1$ the assigned exit track.

- $D_{\text {in }} \in[0,20 \mathrm{~min}]$ the time delay at the entry point.

- $Z_{i}$ where $i=1,2, \ldots, N_{x}-1$ binary parameter characterizing the flight altitude profile $Z_{i}=\left(F L_{i}-F L_{i-1}\right) / 10=$ $\left\{\begin{array}{c}1 \text { if the flight climbs to the next level } \\ 0 \quad \text { at waypoint } \mathrm{i} \\ \text { with }\left(Z_{1}=0\right) .\end{array}\right.$

As our objective is to ensure conflict-free trajectory for each aircraft, we allocate alternative parameters, for each flight, while remaining as close as possible to the initial ones. In addition, another important goal is to benefit from windoptimal routes. For this reason, we intend to take advantages from the jet streams and we propose to use a new wind-optimal route structure instead of the OTS system.

\section{Proposed NeW Route StRUCTURE}

In this section, we propose a new route structure over the NAT airspace which benefits from jet streams. Two major factors influenced the construction of this new structure. First, it is obvious that en-route fuel consumption is strongly influenced by weather conditions, such as wind speed and direction. For this reason, we put the eastbound flights in jet streams direction. The second important factor is that, as mentioned previously, we consider the hypothesis that all aircraft implement the ADS-B system. Therefore, our proposed structure benefits from the reduction of separation norms.

\section{A. Route structure}

As we have seen, in the OTS structure, tracks are separated by $60 \mathrm{NM}$, while, in our new structure, we keep tracks separated by $10 \mathrm{NM}$. This reduction is reasonable since we are interested in aircraft equipped with ADS-B system. Our new structure of routes is described as follow: We keep the same entry and exit points of the OTS tracks. Beginning from the first points of each track, we merge all tracks to the center where we have jet streams. Then, we keep tracks parallel and separated by $10 \mathrm{NM}$ along 1000 NM. Finally, each track joins the corresponding exit point of the OTS system (Figure 3). Besides, in the portion of our structure where tracks are separated by $10 \mathrm{Nm}$, we do not allow aircraft to change their track. Indeed, an aircraft entering in the parallel track section has to keep its track up to the exit point of this section. Obviously, flights crossing the OTS track system do

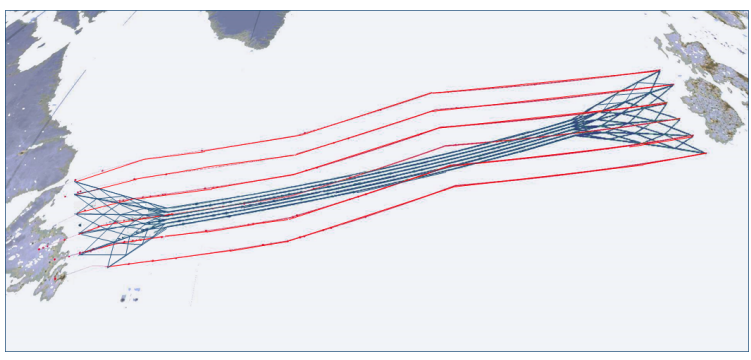

Fig. 3: Superposition of the OTS with the new route structure

not keep the same track from the entering to the exit point. For this reason, our structure has to satisfy this constraint and guarantee reliable transition between the rails. Thus, sections before and after the parallel track region are considered as filters. In these sections each track contains waypoints. Flights are allowed to change their tracks only on these waypoints. Besides, maintaining the preferred flights altitude profile is recommended since it guarantees optimal fuel consumption. Thus, even though we forbid transitions from one track to another inside the parallel track region, aircraft can change their flight levels according to their preferred altitude profiles over this region. For this reason, three waypoints per track are used only to change flight levels while maintaining the same track in the parallel region. Therefore, tracks are represented by a set of waypoints related by links. Flights crossing this route structure are only allowed to change their tracks on 
waypoints (in the filter regions). In each waypoint, the flight has three alternative maneuvers: whether it continues with the same track or it changes its track to an adjacent one (north or south).

\section{B. Route structure model}

We can model our route structure as following. Our structure can be represented as a grid with $N_{y}$ tracks, each track contains $N_{x}$ waypoints and $N_{z}$ flight levels. Figure 4 illustrates the grid model in horizontal dimension. When an aircraft enters a predefined track at a predefined flight level, it's required to follow the same track and flight level unless a maneuver is done. This maneuver can only be held on waypoints. Thus, arriving to a waypoint, a flight has several possibilities to continue its path. It can rather change the flight level or pursue at the same altitude. Besides, when keeping the same flight level, it has also the possibility to change its track to an adjacent one. Thus, only one change is allowed at a given waypoint and changing at the same time the flight level and the track is forbidden. Thus, in addition to the decision variables

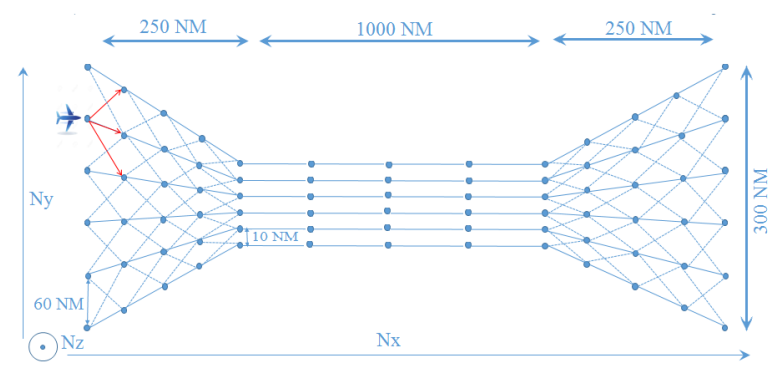

Fig. 4: Horizontal section of the route structure grid model

introduced in the section IV, we introduce the following variables which permit to represent the eventual maneuvers over our new route structure : $X_{i}$ where $i=1,2, \ldots, N_{x}-1$ binary parameter defining the flight routing manoeuvers

$X_{i}=\left\{\begin{array}{cc}1 & \text { if the flight switches to the northern adjacent } \\ \text { track at the waypoint } \mathrm{i}\end{array}\right.$

The $X_{i}$ points represent the waypoints where a rerouting from one track to another is possible, precisely, the waypoints of the filter regions.

\section{CONFlict Detection AND RESOlUtion}

This section presents the methodology used to obtain conflict free trajectories over the NAT airspace. First, we present our conflict detection approach. Then, we describe our conflict resolution algorithm including different ways used to remove conflicts.

\section{A. Conflict detection strategy}

In air traffic control, a conflict represents a violation of established separation norms. As previously mentioned, in our study, we consider oceanic separation norms assuming that all aircraft are equipped with ADS-B systems. For this purpose, we precisely build our route structure (detailed in V-B) based on these reduced separation norms (tracks are separated by 10 NM, flight levels are separated by $1000 \mathrm{ft}$ ). It only remains for us to manage the longitudinal separation which is assumed to be 2 minutes if aircraft are in the same track, and 3 minutes when an aircraft changes its track. Figure 5 shows the longitudinal separation norms. Besides, an aircraft has also the

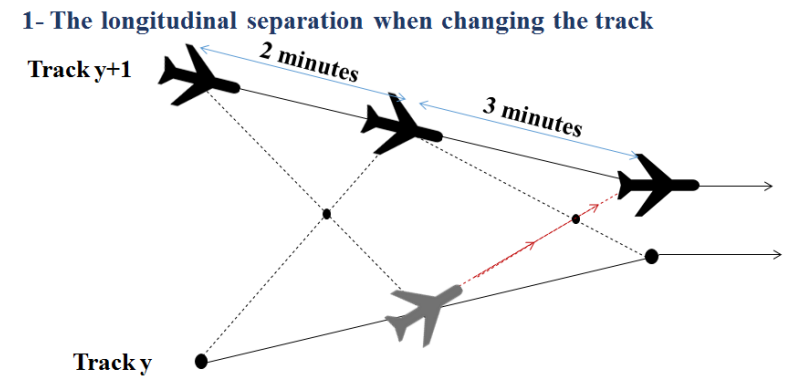

2- The longitudinal separation when changing the flight level

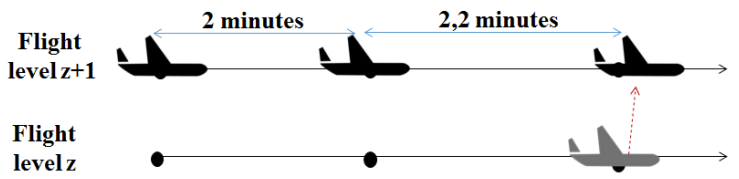

Fig. 5: Longitudinal separation norms

possibility to change its flight level (only by climbing). The aircraft position deviation in the horizontal plane is neglected, as well as the time required to reach the new flight level. However, when changing its flight level, an aircraft has to maintain a new separation norm with aircraft flying on the same track at the new flight level. The separation standards, in this case, become 2,2 minutes. Considering our route structure, we either detect a conflict at nodes or at links. At nodes level, conflicts are detected by sorting flights passing through a given node according to their transit time. Once sorted, we compute the difference in transit time between each two successive flights. A conflict is detected when this value is less than the longitudinal separation. Since each link is delimited by two nodes, we can detect conflict at links level by comparing the sequence order of aircraft at the entry and exit nodes of a link. If there are two swapped flights, then a catch up conflict is detected.

\section{B. Optimization process}

In this work, we seek for optimal flight trajectories by optimizing cruising time while remaining conflict-free and satisfying some constraints such as respecting a maximum delay per flight. To reach this goal, we start with pre-processing the flight set using a sliding window method (SW). The latter consists in dividing the problem into a set of sub-problems. Then, each sub-problem is treated separately and sequentially via a simulated annealing algorithm (SA). The SW method 
consists of sorting the flight set according to the entry time to the route structure. Then, a time window interval that begins at the earliest entry time and with a length $T_{w}$ is fixed. In each time window, four types of flight are defined. Planned flights are those that begin after the time window. Completed flights are those that have already finished their travel time before the time window. Besides, on-going flights are aircraft that begin before the time window and still operate in the considered time interval. Finally, active flights are those having entry time in the considered time window. Once we define the four flight types, we proceed with the de-confliction method. Planned and completed flights are not considered. We apply the SA algorithm to fix the decision variables (parameters) of the active flights while considering the on-going trajectories as constraints. Hence, the decision variables of on-going aircraft are not modified. In the next iteration, we shift the time window by $T_{s}$ (with $T_{s}<T_{w}$ ). This process is repeated until finally all flights are completed. Figure 6 illustrates a schematic example of the SW process. Once a time window

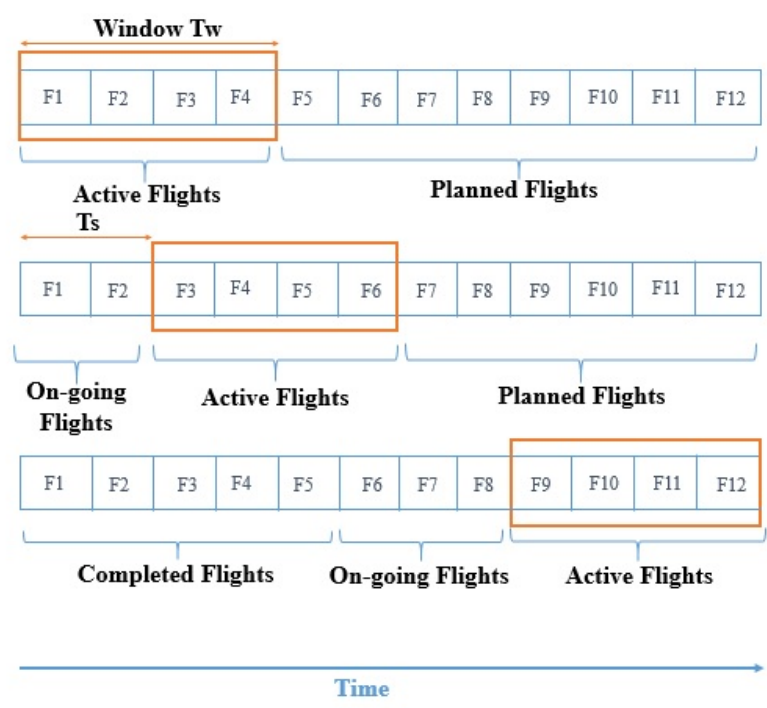

Fig. 6: Sliding window algorithm

is defined with the on-going flight fixed and the activate flight determined, we apply the SA algorithm. The latter is a metaheuristic algorithm inspired from thermodynamics. Further details about this meta-heuristic are present in [16, 17]. SA aims to minimize an energy function. The concept consists in accepting change even to a worse situation, but in a controlled manner. The system begins with a random solution and with a pre-determined control parameter $\mathrm{T}$ (called temperature). The latter decreases as the number of iteration rises. At each step, SA calculates a neighboring solution. It associates to each solution an energy value and probabilistically decides to keep either the neighboring solution or the current one. When $\mathrm{T}$ is large (the heating up step), exploration of the search space is promoted. However, when $\mathrm{T}$ is small (cooling-down step), the system will converge towards the locally best solution. The $\mathrm{SA}$ algorithm is adapted to solve our problem as following:
- The search space consists of all possible set of flight trajectories. A solution is determined when we fix for each aircraft in the flight set the decision variables.

- The energy function is our optimization problem objective function, which we detail in the next section.

- A neighbor solution is obtained by applying a local change to the current solution. It consists of changing one decision variable of an aircraft inside the flight set. The process of getting a neighbor solution is divided in two steps. First, we select the flight to be modified based on the following heuristic. We choose the flight that generates the biggest number of conflicts. Then, we select the decision variable. In order to satisfy as much as possible aircraft preferences, we consider a priority order when modifying flights parameters. Indeed, we give the biggest probability to the shifting of the en-route maneuver, since it does not affect much trajectory length. Then, we consider modifying the entry/exit tracks. If a conflict-free solution does not exist, we delay the flight entry time. The last maneuver is to change the flight level (compared to the one requested by the aircraft). This maneuver is to be considered as a last resort as it involves an increase in the fuel consumption.

- The probability of acceptance: let $s$ be the current solution with $E(s)$ its energy value and let $s^{\prime}$ be a neighboring solution of $s$ with $E\left(s^{\prime}\right)$ its energy value. The acceptance probability of solution $s^{\prime}$ is given by $e^{\left(E(s)-E\left(s^{\prime}\right)\right) / T}$, where $T$ is the temperature.

- The temperature decreases via a geometrical law given by $T_{i}=\alpha * T_{i-1}$.

- The process stops when the temperature $T$ goes below a predefined final temperature, namely $T_{f}$. $T_{f}$ is adjusted to be : $T_{f}=\beta * T_{0}$.

1) Objective function: Our goal is to generate a set of $\mathrm{N}$ eastbound flights optimal trajectories while satisfying several constraints. There are different route optimality criteria such as total trajectories length, flights duration or fuel consumption. In this study, we choose to minimize the total trajectory duration since it is directly related to fuel consumption. Besides, although we have relaxed several flight parameters, our proposed solution must stay as close as possible to airlines preferences. Thus, we add the total delay and the deviation from the desired rail cost to the objective function. The objective function is then the sum of the three following criteria:

- C : Total cruise time

- D : Total entry delay

- R : Total delay induced by the deviation from the requested track.

Cruising time for each flight is the time needed to fly the sum of distance between the crossing waypoints. Thus, total cruise time, $\mathrm{C}$, corresponds to the sum of cruising times over all flights. Total entry delay, D, is calculated in minutes. It represents the sum of entry delays over all flights. The deviation delay related to each flight represents required additional time 
to deviate from the desired track and reach the new assigned one. We get total deviation delay, $\mathrm{R}$, by summing up deviation delays related to all flights. The objective function, that we aim to minimize, is the weighted sum of these values as depicted in:

$$
F_{o b j}=d * D+c * C+r * R
$$

where the non-negative coefficients $(\mathrm{d}, \mathrm{c}, \mathrm{r})$ are used to balance the three criteria up on the user preferences. By minimizing such an objective function, we may obtain a set of optimal trajectories however a conflict-free solution may not exist. For that reason, we have to add the number of induced conflicts to the objective function as the most important criterion to minimize. Actually, we aim to reduce conflicts number to be equal to zero. The objective function becomes then:

$$
F_{o b j}=C_{t}+a *(d * D+c * C+r * R)
$$

where the coefficient (a) is added in order to give the highest priority to the conflict-free criterion. Once all conflicts are resolved, the system starts to minimize the other criteria (cruising time, deviations and delays) while ensuring that the considered solution remain conflict-free.

\section{RESULTS}

In this section, we present our experimental settings and computational results in order to validate our proposed methodology that aim to optimize the strategic eastbound flight planning over NAT airspace. As mentioned earlier, due to the lack of surveillance means, air traffic controllers prefer to keep aircraft in the same track for the entire oceanic route. Since controllers deny re-routing inside the OTS structure, flights exit this structure far from their destinations. This implies the appearance of a very congested zone with a big number of conflicts downstream OTS structure. In order to alleviate the workload of continental controllers, we favor the re-routing of flights inside our proposed route structure so that they exit as close as possible from their destinations. Thus, in the real traffic data we practically find the same entry and exit track for each flight. However, in our simulation tests, we keep the real flight plan, namely: real velocities, entry/exit flight level, entry time and entry track. Then, we generate randomly new exit tracks in order to simulate exit points close to the real aircraft destinations. First, we perform simulations on two real traffic days over the NAT airspace (3rd and 4th August, 2006). Each flight set contains respectively 331 and 378 flights. For these simulations, The parameter values adressed to specify the optimization problem are as follows:

- The initial temperature $T_{0}=0,01$

- The objective function coefficients $a=0,5$ and $d=c=$ $r=1$,

- Number of iteration in each temperature schedule $N=$ 500 ,

- The ratio of the temperature decreasing $\alpha=0,95$,

- The stopping criterion $\beta=0,0001$,

- The sliding window parameters in minutes: $T_{w}=120$, $T_{s}=30$.
TABLE I: Conflict resolution results on two real traffic days

\begin{tabular}{|c|c|c|c|}
\hline \multicolumn{2}{|c|}{ Test } & $03 / 08 / 2006$ & $04 / 08 / 2006$ \\
\hline \multicolumn{2}{|c|}{ Number of flights } & 331 & 378 \\
\hline \multirow{2}{*}{ Number of conflicts } & Before & 1055 & 1548 \\
\cline { 2 - 4 } & After & 0 & 1 \\
\hline \multicolumn{2}{|c|}{ CPU Time (minutes) } & 43 & 50 \\
\hline $\begin{array}{c}\text { Number of flights changing their } \\
\text { vertical profile }\end{array}$ & 69 & 78 \\
\hline
\end{tabular}

TABLE II: Conflict resolution results with different SA configurations

\begin{tabular}{|c|c|c|c|c|}
\hline$\alpha$ & $\mathrm{N}$ & $\begin{array}{c}\text { Number of } \\
\text { conflict }\end{array}$ & $\begin{array}{c}\text { CPU Time } \\
\text { (minutes) }\end{array}$ & $\begin{array}{c}\text { Number of flights } \\
\text { changing their } \\
\text { vertical profile }\end{array}$ \\
\hline \multirow{2}{*}{0,95} & 500 & 119 & 77,6 & 440 \\
\cline { 2 - 5 } & 1000 & 63 & 108,6 & 375 \\
\hline \multirow{2}{*}{0,99} & 500 & 46 & 190,4 & 268 \\
\cline { 2 - 5 } & 1000 & 32 & 398,8 & 200 \\
\hline
\end{tabular}

Actually, the parameters of the SA and SW algorithm are empirically set. On the other side, we adjust the objective function parameters in order to give more priority to the reduction of the number of conflicts, thus we set $a=0,5$. Besides, we give equal priorities to the other different creterion, namely total cruise time, total delay and total track shift. Hence, we set $d=c=r=1$. The simulation results for both studied flight sets are presented in table I.

As we can see in table I, we conclude that the SA and SW algorithm found almost conflict-free solutions for the two studied flight sets with a reasonable CPU time which is about 50 minutes. Concerning the second flight set (4th August), we denote that 1 residual conflict is unsolved. This remaining conflict can be solved by setting the parameter $a$ of the objective function to 0,1 . Thus, we give a bigger priority to conflict number reduction rather than the other criteria. Furthermore, we evaluate the number of flights that changes their vertical profile. In each flight set, typically 70 flight changes their optimal step climbs, thus the rest of the flight set keeps the vertical profile that ensures an optimal fuel consumption. Besides, we evaluate the delay induced for the conflict-free solution. We notice that about 300 flight in each set are within 10 minutes of delay and only some flights are approaching the limit delay which is 20 minutes. These encouraging results allowed us to expend the flight number and re-experiment the simulations. Thus, we generate randomly a flight set containing 1000 flights. Initially, the considered flight set generates 5501 conflicts. After applying SA and SW algorithm, we noticed a significant number reduction that reaches 119 conflicts. However, a conflict-free solution does not exist with the previously mentioned configuration of the SA algorithm. For this reason, we tested our problem with different SA algorithm configurations. Table II summarizes the obtained results. We notice that the number of remaining conflict, as well as The number of flights that changes their requested vertical profile, are strongly affected by the changing of both parameters $\alpha$ and $N$. Slowly lowering the temperature, by decreasing the parameter $\alpha$, is influencing more the results. 
Though, more CPU time is needed to get an optimal solution. Nevertheless, although the search space is further explored by increasing the parameters $\alpha$ and $N$, no conflict free solution is found. Conflict number decreases from 5501 to 32. This is considered, in our case, as a very interesting result. In fact, we are tackling a strategic conflict resolution, and therefore, any remaining conflicts could be resolved in pre-tactical and tactical phases.

\section{CONCLUSION}

In this paper, a new route structure over the NAT airspace is proposed. The main idea is to benefit from jet streams in order to construct trajectories that are as close as possible to the wind optimal flight paths. Assuming that all aircraft are equipped with ADS-B systems, it is possible to consider reduced separation norms between aircraft operation on NAT airspace. Then, an optimization algorithm that aim to resolve potential conflict over the route structure while generating almost wind optimal trajectories has been developed.

Simulations were conducted on different flight sets. First, we perform simulation for two real traffic data over the NAT airspace containing 331 and 378 flights. Then, we extend the flight number and we generate randomly a flight set containing 1000 flights. The computational results show that a significant reduction of the number of conflicts can be reached. Since we are performing strategic conflict resolution, the remaining number of conflict can be resolved further in tactical or pretactical stage.

In future works, we are planning to use real jet streams to design route structure, and therefore compare the optimized flight trajectories with the actual ones followed by aircraft, namely using the OTS routes. Besides, we are planning to take into account the fuel consumption, and thus evaluate potential gain estimated by applying the proposed wind-optimal route structure. We also plan to compare our results with those of other optimization algorithms that address our problem.

\section{REFERENCES}

[1] NAT Doc 007, "NORTH ATLANTIC OPERATIONS AND AIRSPACE MANUAL". International Civil Aviation Organization (ICAO) European and North Atlantic (EUR/NAT) Office, 2016.

[2] Dean C. Miller William R. Richards, Kathleen O'Brien. New Air Traffic Surveillance Technology. Avionics and Air Traffic Management, 2010.

[3] H. Blomenhofer, A. Pawlitzki, P. Rosenthal, and L. Escudero. Space-based automatic dependent surveillance broadcast (ads-b) payload for in-orbit demonstration. In 2012 6th Advanced Satellite Multimedia Systems Conference (ASMS) and 12th Signal Processing for Space Communications Workshop (SPSC), pages 160-165, Sept 2012.

[4] Benefits of a global space-based ads-b surveillance system. In 2014 Integrated Communications, Navigation and Surveillance Conference (ICNS) Conference Proceedings, pages 1-15, April 2014.
[5] O. Rodionova, M. Sbihi, D. Delahaye, and M. Mongeau. North atlantic aircraft trajectory optimization. IEEE Transactions on Intelligent Transportation Systems, 15(5):2202-2212, Oct 2014.

[6] Olga Rodionova, Mohammed Sbihi, Daniel Delahaye, and Marcel Mongeau. Optimization of aicraft trajectories in north atlantic oceanic airspace. In ICRAT 2012, 5th International Conference on Research in Air Transportation, pages pp-xxxx, 2012.

[7] I. Dhief, N. H. Dougui, D. Delahaye, and N. Hamdi. Strategic planning of aircraft trajectories in north atlantic oceanic airspace based on flocking behaviour. In 2016 IEEE Congress on Evolutionary Computation (CEC), pages 2438-2445, July 2016.

[8] Brunilde Girardet, Laurent Lapasset, Daniel Delahaye, and Christophe Rabut. Wind-optimal path planning: Application to aircraft trajectories. In Control Automation Robotics \& Vision (ICARCV), 2014 13th International Conference on, pages 1403-1408. IEEE, 2014.

[9] Roberto F Patron, Aniss Kessaci, Ruxandra Mihaela Botez, and Dominique Labour. Flight trajectories optimization under the influence of winds using genetic algorithms. In AIAA Guidance, Navigation, and Control (GNC) Conference, page 4620, 2013.

[10] Banavar Sridhar, Hok K Ng, Florian Linke, and Neil Y Chen. Benefits analysis of wind-optimal operations for trans-atlantic flights. In 14th AIAA Aviation Technology, Integration, and Operations Conference (ATIO), Atlanta, $G A, 2014$.

[11] H. K. Ng, B. Sridhar, and S. Grabbe. A practical approach for optimizing aircraft trajectories in winds. In 2012 IEEE/AIAA 31st Digital Avionics Systems Conference (DASC), pages 3D6-1-3D6-14, Oct 2012.

[12] Banavar Sridhar, Neil Y Chen, K Ng Hok, Olga Rodionova, Daniel Delahaye, and Florian Linke. Strategic planning of efficient oceanic flights. In ATM seminar 2015, 11th USA/EUROPE Air Traffic Management R\&D Seminar, 2015.

[13] Olga Rodionova, Daniel Delahaye, Banavar Sridhar, and Hok K Ng. Deconflicting wind-optimal aircraft trajectories in north atlantic oceanic airspace. In AEGATS '16, Advanced Aircraft Efficiency in a Global Air Transport System, 2016.

[14] Shon Grabbe, Banavar Sridhar, and Nadia Cheng. Central east pacific flight routing. Air Traffic Control Quarterly, 15(3):239-264, 2007.

[15] Shon Grabbe, Banavar Sridhar, and Avijit Mukherjee. Central east pacific flight scheduling. In AIAA Guidance, Navigation, and Control Conference and Exhibition, Hilton Head, South Carolina, 2007.

[16] Chii-Ruey Hwang. Simulated annealing: Theory and applications. Acta Applicandae Mathematica, 12(1):108111, 1988.

[17] Scott Kirkpatrick, C Daniel Gelatt, Mario P Vecchi, et al. Optimization by simmulated annealing. science, 220(4598):671-680, 1983. 